\title{
Flame Retardancy of Polysiloxane Solid Powder Combined with Organophosphate in Polycarbonate
}

\author{
Wenjun ZHOU ${ }^{\mathrm{a}}$, Ke CHEN ${ }^{\mathrm{b}}$ and Weizhuang $\mathrm{HE}^{\mathrm{c}}$ \\ College of Materials, Chemistry and Chemical Engineering, Hangzhou Normal University, Hangzhou \\ 310036, China \\ acjhzwj@163.com, byiyefeng@yahoo.cn, c315812087@qq.com
}

\begin{abstract}
The synergistic effects of methylphenyl-polsiloxane solid powder and organophosphate flame retardant for polycarbonate (PC) were investigated by limiting oxygen index(LOI), and cone calorimeter. It was found that the LOI of the flame-retarded PC (FR-PC) with polysiloxane and organophosphate binary flame retardants didn't increase in comparison with that of FR-PC containing a single species flame retardant. The cone calorimeter results show that the heat release rate (HRR) of the $\mathrm{PC} /$ polysiloxane/organophosphate(PX230) composition is approximately $50 \%$ lower than that of the PC/polysiloxane or the PC/PX230. Total heat release (THR) of PC/polysiloxane/PX230 also significantly reduce in comparison with that of PC/polysiloxane and PC/PX230 binary composites, which indicates a clear synergistic effect of the polysiloxane and PX230 in the PC flame retardant composites. The $\mathrm{CO}$ production rate of the $\mathrm{PC} /$ polysiloxane/PX230 during combustion is slightly lower than that of the virgin PC. So the combination of the polysiloxane with PX230 used in PC could synergic inhibit the combustion heat release, and achieve environmental friendly fire-retardant effect.
\end{abstract}

Keywords: polycarbonate, polysiloxane, organophosphate, flame retardant, synergistic effect

\section{Introduction}

Bisphenol A polycarbonate(PC), an engineering plastics with excellent impact strength, heat resistance, dimensional stability, and transparency is widely used in such diverse applications as automotive components, electric and electronic appliances, building fields, and office automation. PC itself exhibits flame retardancy, and its limiting oxygen index (LOI) is about $26 \%$. However, flame retardant technique must be applied to PC in order to satisfy the stringent fire retardant requirements of electrical and electronic device applications. Organic halogen compounds have been commonly used for PC flame retarding because of their extremely high flame retardant efficiency. However, these compounds might generate highly toxic and potentially carcinogenic substance during combustion. So from an environmental standpoint, it is preferable to develop flame retardants that do not contain 
halogens. Various halogen-free flame retardants have been tried to use in polymers in recent years [1-2].

Efforts have been made on the technique of synthesizing new flame retardants with no or less amount of toxic substances forming during combustion and with high performance[3-4]. Simultaneously, approaches have been made to combine different flame retardants or flame retardants with additives, respectively, and thus to obtain superior synergisms in flame retardancy[5-6]. Pawlowski et al studied flame retardancy of aryl phosphates in combination with boehmite (ALOOH) [7] and with zinc borate[8] in PC/ acrylonitrile-butadiene-styrene (ABS) blends. It was concluded that adding ALOOH can significantly enhance barrier effects of the fire residue during burning. They also found combination zinc borate and BDP exhibit a clear synergy in LOI.

Organophosphates, such as triphenyl phosphate (TPP), resorcinol bis(diphenyl phosphate) (RDP), BDP are appropriate halogen-free flame retardants being widely used in PC and PC-based blends. It has been reported that phosphates form volatile PO, P and $\mathrm{P}_{2}$ species during combustion that act in the gas phase through flame inhibition. The fire retardant action of phosphates could also take place in the condensed phase, and this condensed phase mechanism results from char formation through esterification and dehydration[9].

Polysiloxanes have high heat resistance, generating no harmful gases during burning, and furthermore polysiloxanes themselves are highly safe, and are increasing used for improving flame retardancy of polymers, especially for PC and PC-based blends[10]. Polysiloxane is polymer including one or more of the following four siloxane units: $\mathrm{M}$ units (R3SiO0.5), D units (R2SiO1.0), T units(RSiO1.5), and Q units (SiO2.0). Flame-retardancy of polysiloxane is closely related to its structure (siloxane unit and Organic side groups) [11-12]. Polysiloxane only with a specific structure has good flame retardancy. In addition, most existing polysiloxane flame retardants are liquid form and are difficult for the subsequent complex processing if they are applied to flame retardant polymers. In this paper we have synthesized a kind of methylphenyl-polysiloxane solid powder with good flame retardancy through co-hydrolysis condensation reaction using tetraethoxysilane (TEOS) as the raw materials to introduce an appropriate amount of $\mathrm{Q}$ units in polysiloxane. The polysiloxane powder was used together with organophosphates in an effort to achieve a better flame-retardant effect for PC. The synergistic effect of the polysiloxane and organophosphates in flame-retarded PC systems was evaluated by limiting oxygen index (LOI) test, and cone calorimeter.

\section{Experimental}

\subsection{Materials}

Polycarbonate (PC, CALIBRE 201-10) was purchased from LG-DOW Polycarbonate Ltd, Korea. Triphenyl phosphate (TPP) was produced by Shanghai Juhao Fine Chemical Co., Ltd, China. Resorcinol bis( diphenyl phosphate) (RDP) was produced by Supresta LLC, USA. PX-230, a high-molecular-weight halogen-free organophosphate flame retardant solid powder (P content: above 10.5\%) , was purchased from Shanghai Dynamo Chemical Co., Ltd, China.

Methylphenyl-polysiloxane solid powder to be tested was systhesized through co-hydrolysis condensation reaction using TEOS and methoxysilane compounds as raw materials[13]. The Methylphenyl-polysiloxane solid powder consisted of $25 \mathrm{~mol} \%$ Q units, 
and $75 \mathrm{~mol} \%$ phenyl in its organic groups. Its end groups are mainly methyl with a few hydroxyls. The ratio of organic groups to silicon atoms $(\mathrm{R} / \mathrm{Si})$ was 0.8 .

The $\mathrm{PC}$ was dried in the oven at $102^{\circ} \mathrm{C}$ for $24 \mathrm{~h}$ prior to blending. The flame-retarded $\mathrm{PC}$ (FR-PC) sample was blended with $95 \mathrm{wt} \%$ of the PC substrate and $5 \mathrm{wt} \%$ of the total flame retardants by melt blending in a XSS-300 torque rheometer at a temperature of $260^{\circ} \mathrm{C}$, Then the samples were molded into test specimens in a TY-200 injection molding machine at an injection temperature of $285^{\circ} \mathrm{C}$. The details of their compositions are shown in Table 1.

TABLE 1 FORMULATIONS OF THE FLAME-RETARDED PC COMPOSITES

\begin{tabular}{cccc}
\hline Sample no. & PC $(w t \%)$ & Polysiloxane $(w t \%)$ & Organophosphate* $(w t \%)$ \\
\hline FRPC-1 & 95 & 5 & 0 \\
FRPC-2 & 95 & 4.95 & 0.05 \\
FRPC-3 & 95 & 4.5 & 0.5 \\
FRPC-4 & 95 & 4 & 1 \\
FRPC-5 & 95 & 2.5 & 2.5 \\
FRPC-6 & 95 & 1 & 4 \\
FRPC-7 & 95 & 0.5 & 4.5 \\
FRPC-8 & 95 & 0.05 & 4.95 \\
FRPC-9 & 95 & 0 & 5 \\
\hline \multicolumn{4}{c}{ * Organophosphate can be TPP, or RDP, or PX-230. }
\end{tabular}

\subsection{Characterization}

The LOI value was measured on an oxygen index instrument XZT-100 produced by Dahua Chengde Testing Machine Co., China, according to the standard oxygen index test ASTM D2863. A cone calorimeter (Stanton Redcroft, FTT, UK )was used to characterize the forced flaming behaviour of the PC and FR-PC samples according to ISO 5660. The sample $(100 \mathrm{~mm} \times 100 \mathrm{~mm} \times 4 \mathrm{~mm})$ was wrapped in an aluminum foil and exposed horizontally to the external heat flux of $50 \mathrm{~kW} / \mathrm{m}^{2}$.

\section{Results and Discussion}

\subsection{Flame retardancy by LOI test}

The flame retardant compounds, which are the methylphenyl-polysiloxane solid powder combined with three different organophosphate flame retardants RDP, TPP, PX230, respectively, were used for PC in accordance with the formula showed in Table 1 . The LOI results for PC/polysiloxane/RDP, PC/polysiloxane/TPP, and PC/polysiloxane/PX-230 are given in Figs. 1, 2, and 3, respectively. In all the flame retardant composite systems, the flame retardant PC (FR-PC) adding the polysiloxane alone achieves the best flame retardancy. The LOI of $95 \mathrm{wt} \% \mathrm{PC} / 5 \mathrm{wt} \%$ polysiloxane is $33.3 \%$ that is $28.1 \%$ higher than virgin PC's LOI of $26 \%$. PX230 has the best flame retardant efficiency among the organophosphate flame retardants to be tested. The LOI of $95 \mathrm{wt} \% \mathrm{PC} / 5 \mathrm{wt} \% \mathrm{PX} 230$ is $32.2 \%$, whereas the LOI of $95 \mathrm{wt} \% \mathrm{PC} / 5 \mathrm{wt} \% \mathrm{RDP}$ and $95 \mathrm{wt} \% \mathrm{PC} / 5 \mathrm{wt} \% \mathrm{TPP}$ are all $30.2 \%$. 


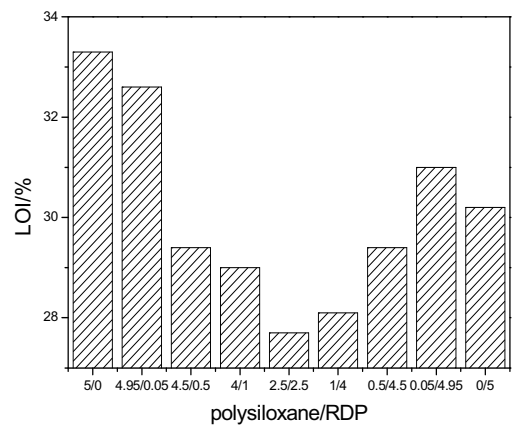

Fig.1. LOI data of the PC/polysiloxane/RDP

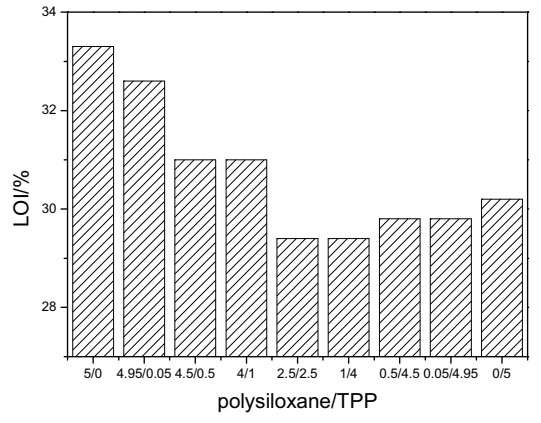

Fig.2. LOI data of the PC/polysiloxane/TPP

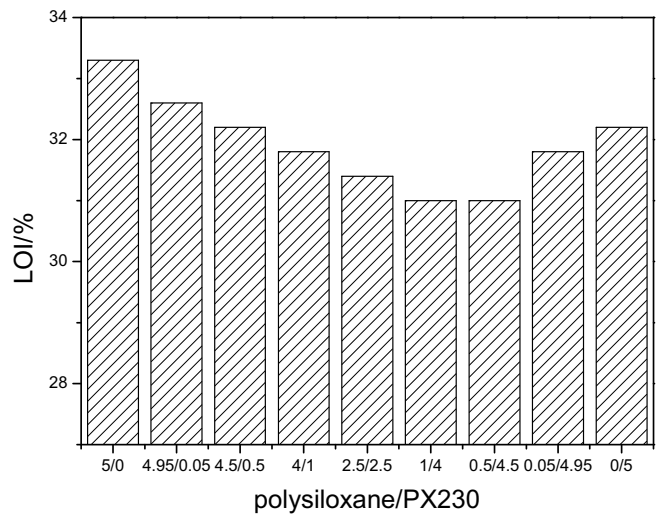

Fig.3. LOI data of the PC/polysiloxane/PX230

When the content of the polysiloxane in the flame retardant composite is higher than that of the organophosphate (RDP, or TPP, or PX230), namely when the polysiloxane solid powder is the main flame retardant, the LOI of the FR-PC decreases with the increase of the content of the organophosphate flame retardant. As shown in Fig.1, when the content of the $\mathrm{RDP}$ in the flame retardant composite is higher than that of the polysiloxane, namely when the RDP is the main flame retardant, the LOI of the FR-PC increases first and then decreases with the increase of the content of polysiloxane. The main flame retardant action of RDP in the condensed phase results from char formation through esterification and dehydration wherein phosphorus compounds act like acid precursors[9]. Adding a small amount of the polysiloxane to the $\mathrm{PC} / \mathrm{RDP}$ promotes the formation of char, and the LOI increases. However, slightly more polysiloxane content makes the LOI of PC/RDP decrease. From Fig. 2 and 3, it can be seen that the trend of the LOI of PC/polysiloxane/TPP is very similar to $\mathrm{PC} /$ polysiloxane/PX230. When the content of PX230 in the flame retardant composite is higher than that of the polysiloxane, the LOI of the PC/polysiloxane/PX230 decreases with the increase of the content of the polysiloxane, and reaches the minimum $(31.0 \%)$ when the ratio of polysiloxane/PX230 is $1 / 4$ and $0.5 / 4.5$. In general, the LOI of the FR-PC with combination of polysiloxane and organophosphate is poorer than that of the FR-PC with each individual flame retardant, especially compared with the individual polysiloxane. It is concluded that the polysiloxane and organophosphate (RDP, or TPP, or PX230) works antagonistically with respect to the LOI for PC. 
In order to further understand fire behavior and interpret the variation of the LOI of the PC/polysiloxane/ phosphate, flame and char layer of the FR-PC samples in LOI test was observed carefully. Fig. 4 shows a comparison of char layer after LOI test for PC/polysiloxane, PC/PX230 and PC/polysioxane/PX230. As reported in an earlier study[14], the main flame retardant action of polysiloxane is in the condensed phase. The methylphenyl-polysiloxane could induce cross-linking reactions in PC/polysiloxane system, and promote to form char containing silicon on the surface of the FR-PC during combustion. From Fig. 4(a), it can be seen that the abundant protective char layer forming during the $\mathrm{PC} /$ polysiloxane combustion completely covered above the PC resin without dripping phenomenon. The protective char layer can restrain the diffusion of flammable degradation products into the combustion zone and keep the underlying polymer away from the flame. Thus the LOI of the PC significantly improved.

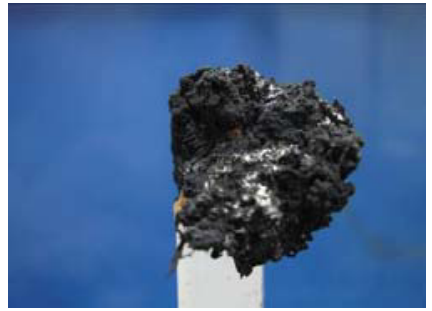

(a)

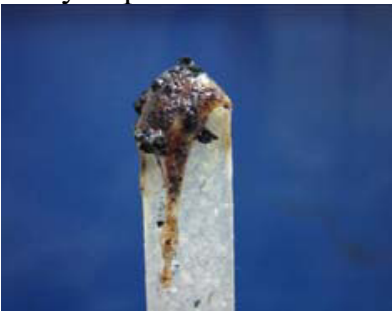

(b)

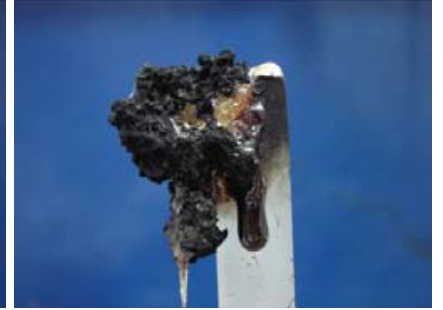

(c)

Fig. 4 Char layer of the samples after LOI test: (a) $95 \% \mathrm{PC} / 5 \%$ polysiloxane, (b) $95 \% \mathrm{PC} / 5 \% \mathrm{PX} 230$, and (c) $95 \% \mathrm{PC} / 2.5 \%$ polysiloxane $/ 2.5 \% \mathrm{PX} 230$

The fire retardant action of PX230, as an organic phosphate flame retardant, could take place in the condensed phase due to char formation through esterification and dehydration. On the other hand, phosphates could form volatile PO, $\mathrm{P}$ and $\mathrm{P}_{2}$ species during combustion that act in the gas phase through flame inhibition [9]. Visually, low intensity flame accompanied the PC/PX230 burning over the sample, and the thin char layer formed. As shown in Fig. 4(b), compared to the PC/polysiloxane, the PC/PX230 sample combustion formed less char with dripping to some extent. It was observed that the intensity of the flame was low and the flame was easy to put out. Thus, although the PC/PX230 blend formed less char during combustion, because of the role of flame suppression of PX230 made the LOI of the blend effectively increase.

From Fig.4(c), the abundant char layer can be seen after LOI test of the $\mathrm{PC} /$ polysiloxane/PX230 blend. The polysiloxane's action in the promotion of charring is obvious, which is similar to the PC/polysiloxane system. However, due to the role of PX230 on making melting drop during combustion, large amount of char which was overweight dripped more seriously by gravity, and part of the polymer resin surface directly exposed to flame without the protection of char layer. It caused the LOI of the PC/polysiloxane/PX230 decrease.

As the PX230 has the best flame retardant efficiency among all organophosphates to be tested, the PX230 was selected to investigate the synergistic effect in PC/polysiloxane flame retardant system in subsequent work.

\subsection{Cone calorimeter study}

The cone calorimeter tests were used to evaluate the fire performance of the PC/polysiloxane/PX230 system. Figs. 5 and 6 show a comparison of heat release rate (HRR) and total heat release (THR)curves for the virgin $\mathrm{PC}, 95 \mathrm{wt} \% \mathrm{PC} / 5 \mathrm{wt} \%$ polysiloxane, $95 \mathrm{wt} \% \mathrm{PC} / 5 \mathrm{wt} \% \mathrm{PX} 230,95 \mathrm{wt} \% \mathrm{PC} / 2.5 \mathrm{wt} \%$ polysiloxane $/ 2.5 \mathrm{wt} \% \mathrm{PX} 230$, respectively. The flammability data of virgin PC and the FR-PC from cone calorimeter investigation are 
summarized in Table 2, including time to ignition (TTI), average HRR, peak HRR, THR, and average effective heat of combustion (EHC). The TTI of the FR-PC samples are lower than that of virgin PC, especially the PC/polysiloxane blend obtains the lowest TTI. The reason may be due to the fact that the temperatures of thermal degradation of the FR-PC samples with polysiloxane or PX230 are lower in comparison with virgin $\mathrm{PC}$, and thus before ignition, the FR-PC composites can degrade releasing more volatile flammability compounds, which make ignition easier.

The HRR values of the FR-PC samples are all lower than that of virgin PC, and the HRR value of the PC/polysiloxane/PX230 is comparatively the lowest. The peak HRR and average HRR of the PC are $289.5 \mathrm{~kW} / \mathrm{m}^{2}$ and $180.6 \mathrm{~kW} / \mathrm{m}^{2}$, respectively. Whereas the peak HRR and average HRR of the PC/polysiloxane $/ \mathrm{PX} 230$ composition are $124.8 \mathrm{~kW} / \mathrm{m}^{2}$ and $75.6 \mathrm{~kW} / \mathrm{m}^{2}$, respectively, which are $56.9 \%$ and $58.2 \%$ lower than that of virgin PC. Further, the HRR of the PC/polysiloxane/PX230 composition is approximately 50\% lower than that of the PC/polysiloxane or the PC/PX230, revealing a clear synergistic effect of the polysiloxane and PX230 in HRR. Total heat release (THR) of the PC/polysiloxane/PX230 also significantly reduces in comparison with pure PC and PC containing a single flame retardant.

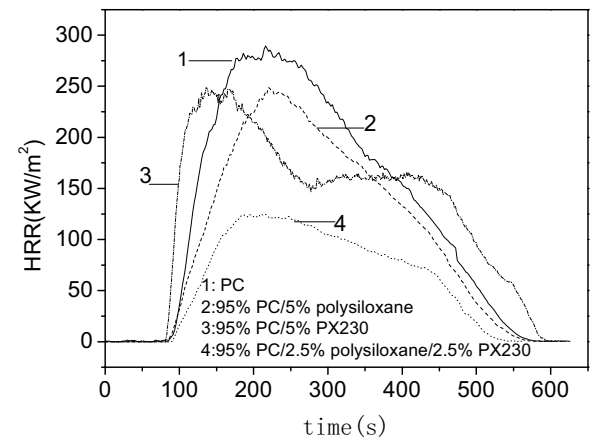

Fig. 5. HRR curves of $\mathrm{PC}$ and flame retardant $\mathrm{PC}$

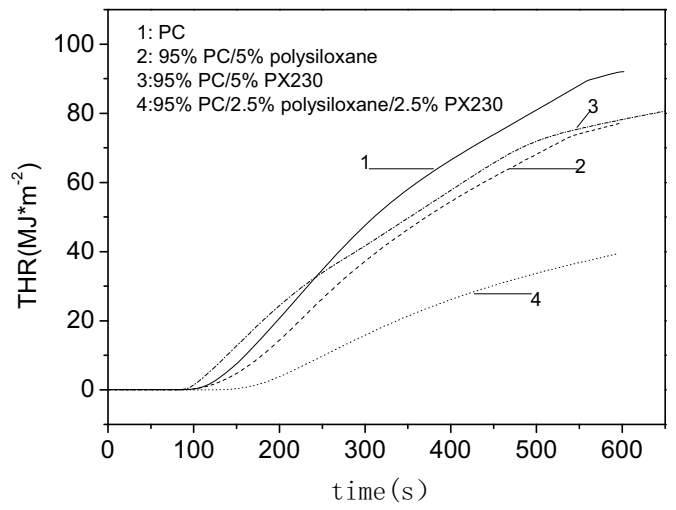

Fig. 6. THR curves of PC and flame retardant PC

The polysiloxane in PC flame retardant system can react with the degradation products of $\mathrm{PC}$ to form cross-linking structure during heating. Simultaneously, the polysiloxane moves to PC surface, and silicon accumulates on the surface of the polymer to form a silicon-rich 
char layer[12]. THR characterizes the fire load and HRR influences the fire growth observed in the cone calorimeter. Both the lowest HRR and THR values of the $\mathrm{PC} /$ polysiloxane/PX230 indicate that the polysiloxane reacts with the phosphate in addition to reaction with the degradation products of the PC to form a silicon-phosphate structure char, which provides more effective barrier action to restrain mass transport and to protect the underlying polymer from the flame. Thus, the flame retardancy of the PC further improves.

Table 2 Cone Calorimetric Data Of Pc And Its Flame Retardant Composites

\begin{tabular}{cccccc}
\hline Sample & TTI(s) & $\begin{array}{c}\text { Average } \\
\text { HRR } \\
\left(\mathrm{kW} / \mathrm{m}^{2}\right)\end{array}$ & $\begin{array}{c}\text { Peak } \\
\mathrm{HRR} \\
\left(\mathrm{kW} / \mathrm{m}^{2}\right)\end{array}$ & $\begin{array}{c}\text { THR } \\
\left(\mathrm{MJ} / \mathrm{m}^{2}\right)\end{array}$ & $\begin{array}{c}\text { Average } \\
\text { EHC } \\
(\mathrm{MJ} / \mathrm{kg})\end{array}$ \\
\hline PC & 84 & 180.6 & 289.5 & 92.0 & 22.48 \\
95\%PC/5\%polysiloxane & 64 & 146.3 & 248.6 & 76.6 & 27.83 \\
$\begin{array}{c}\text { 95\%PC/5\%PX230 } \\
\text { 95\%PC/2.5\%polysiloxane }\end{array}$ & 75 & 147.4 & 248.5 & 77.7 & 17.76 \\
/2.5\%PX230 & 74 & 75.6 & 124.8 & 39.1 & 18.92 \\
\hline
\end{tabular}

The effective heat of combustion (EHC), which is total heat release of the unit of mass loss, is a direct measure for flame inhibition in the gas phase. The comparative analysis of the EHC test results (Table 2) leads to a conclusion that PX230 makes average EHC of the PC reduce, which indicates that PX230 acts through flame inhibition in the gas phase. The organophosphate flame retardant PX230 decomposes in the flame to form several types of phosphorus radicals, such as $\mathrm{PO}$ radicals. These radicals react with the highly reactive $\mathrm{H} \cdot$ or $\mathrm{OH} \cdot$ radicals during combustion, thus the flame is effective inhibited. However, average EHC of $95 \% \mathrm{PC} / 5 \%$ polysiloxane is $27.83 \mathrm{MJ} / \mathrm{kg}$, which is higher than that of pure PC. This result indicates that the polysiloxane has no action of flame inhibition in the gas phase during combustion. The superior flame retarding effect of the polysiloxane for PC results from the formation of the crosslinking structure char during combustion, that is condensed phase flame retardant mechanism. Combining polysiloxane and PX230 in PC results in decrease in average EHC value, whereas the value is higher than that with PX230 alone. The FR-PC with polysiloxane and PX230 composite flame retardant has less phosphorus content, and so less phosphorus species are released for flame inhibition in the gas phase compared to the FR-PC with PX230 alone.

Fig. 7 shows smoke production rate (SPR) curves of the PC and the FR-PC samples. It can be seen that peak smoke production rate of the PC/polysiloxane is the least among all of the samples to be tested. This result is attributes to the action of the polysiloxane in the promotion of charring. The insulation effect of the char layer inhibits the combustion of the materials. However, smoke release of the PC/PX230 or the PC/polysiloxane/PX230 complex system doesn't obviously decline. According to the heat release data, the flame retardant system containing PX230 truly inhibits the heat release during combustion, especially in the $\mathrm{PC} /$ polysiloxane/PX230. It is speculated that there is no significant reduction of smoke by PX230 owing to organophosphate flame retardant changing the thermal degradation process of the PC, allowing part of the material turn a complete combustion into incomplete combustion. Incomplete combustion produces less heat and more smoke.

It is observed that the $\mathrm{CO}$ production rate of the $\mathrm{PC} /$ polysiloxane decreased in the main fire range compared with virgin PC(Fig.8). The results are consistent with the data of smoke production rate. The $\mathrm{CO}$ production rate of the $\mathrm{PC} / \mathrm{PX} 230$ is higher than that of the $\mathrm{PC}$, similar to the smoke release rate. It is worthwhile to note the trend of $\mathrm{CO}$ production of $\mathrm{PC}$ and the FR-PC composites. Compared to virgin $\mathrm{PC}, \mathrm{CO}$ production rate of the $\mathrm{PC} /$ polysiloxane reduces obviously, whereas the PC/PX230 increases. The combination of polysiloxane and PX230 to be added to the PC results in a slight decrease in CO production rate. Therefore, it can be concluded that the combination of the methylphenyl-polysiloxane 
solid powder and PX230 to be used to PC synergic inhibits the combustion heat release, while not enhance the release of harmful gases, and achieves environmental friendly fire-retardant effect.

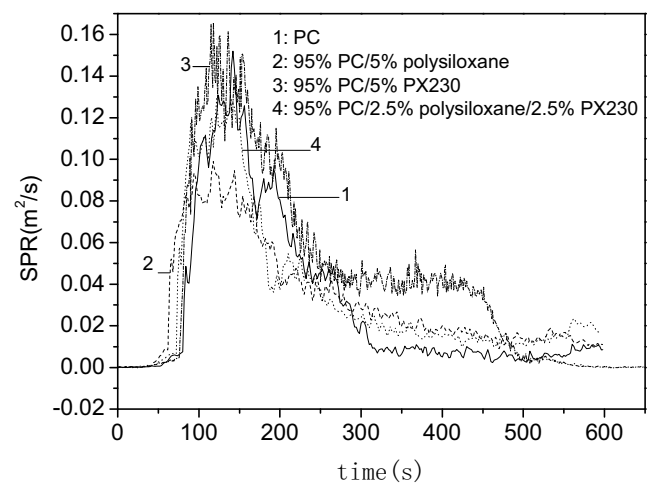

Fig. 7. SPR of PC and flame retardant PC

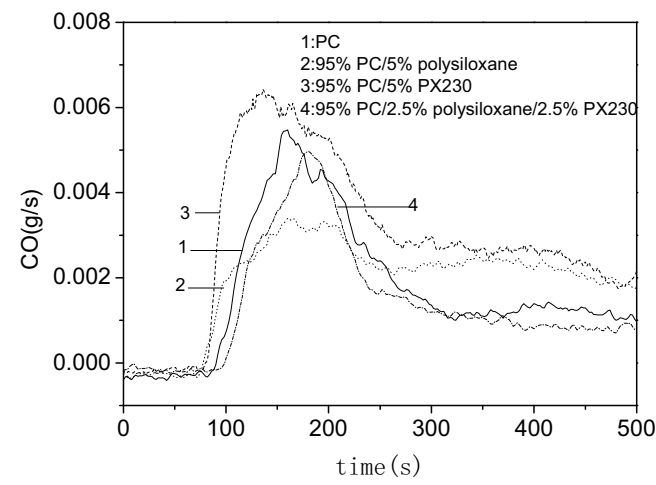

Fig.8. CO production rate of $\mathrm{PC}$ and flame retardant PC

\section{Conclusions}

The flame-retarded PC composites were prepared by melt blending with PC substrate, methylphenyl-polysiloxane solid powder and organophosphate flame retardant. The interaction between polysiloxane and the organophosphate PX230 promotes formation of the char during combustion. However, more char forms during combustion and the role of PX230 on making melting drop render the dripping of PC/ polysiloxane/PX230 more serious by gravity, and part of the polymer resin surface directly exposes to flame without the protection of char layer. Thus, the LOI of PC/polysiloxane/PX230 decreases in comparison with PC/polysiloxane and PC/organophosphate binary composites.

Cone calorimeter tests reveal a clear synergistic effect of the polysiloxane and PX230 in PC composites with respect to HRR. The HRR of PC/polysiloxane/PX230 composition is approximately 50\% lower than that of PC/polysiloxane or PC/PX230. The THR of $\mathrm{PC} /$ polysiloxane/PX230 also significantly reduces in comparison with virgin $\mathrm{PC}$ and the $\mathrm{PC}$ containing a single flame retardant. Compared to virgin $\mathrm{PC}, \mathrm{CO}$ production rate of 
PC/polysiloxane/PX230 slightly decreases. The methylphenyl-polysiloxane solid powder and PX230 composite flame retardant is effective in retarding the combustion of PC.

\section{Acknowledgements}

The work was financially supported by the Natural Science Foundation of Zhejiang Province of China (No. LY14E030009), and the Commonweal Technology Research Project of Zhejiang Province of China (No. 2015C31146).

\section{References}

1. S.V. Levchik, E.D. Weil, Flame Retardants in Commercial Use or in Advanced Development in Polycarbonates and Polycarbonate Blends, J. Fire Sci., 24(2006) 137-151.

2. A. Nodera, Flame Retardancy of a Polycarbonate-Polydimethylsiloxane Block Copolymer: the Effect of the Dimethylsiloxane Block Size, J. Appl. Polym. Sci., 100(2006) 565-575.

3. Z. Tang, Y. Li, Y. J. Zhang, Oligomeric Siloxane Containing Triphenylphosphonium Phosphate as a Novel Flame Retardant for Polycarbonate, Polym. Degrad. Stab., 2012, 97, 638-644.

4. J. Ye, G. Liang, A. Gu, Z. Zhang, Novel Phosphorus-Containing Hyperbranched Polysiloxane and its High Performance Flame Retardant Cyanate Ester Resins, Polym. Degrad. Stab., 98(2013) 597-608.

5. X. Wang, Y. Hu, L. Song, H. Yang, Comparative Study on the Synergistic Effect of POSS and Graphene with Melamine Phosphate on the Flame Retardance of Poly(butylene succinate), Thermochim. Acta, 543 (2012) 156-164.

6. X. Chen, C. Jiao, Synergistic Effects of Hydroxyl Silicone Oil on Intumescent Flame Retardant Polypropylene System, Fire Safety J., 44 (2009) 1010-1014.

7. K.H. Pawlowski, B. Schartel, Flame Retardancy Mechanisms of Aryl Phosphates in Combination with Bboehmite in B Polycarbonate/Acrylonitrile-Butadiene-Styrene Blends, Polym. Degrad. Stab., 93(2008) 657-667.

8. K.H. Pawlowski, B. Schartel, and M.A. Fichera, Flame Retardancy Mechanisms of Bisphenol A Bis(diphenyl phosphate) in Combination with Zinc Borate in Bisphenol A Polycarbonate/Acrylonitrile -Butadiene-Styrene Blends, Thermochim. Acta, 498 (2010) 92-99.

9. K.H. Pawlowski, and B. Schartel, Flame Retardancy Mechanisms of Triphenyl Phosphate, Resorcinol Bis(diphenyl phosphate) and Bisphenol A Bis(diphenyl phosphate) in Bisphenol A Polycarbonate/Acrylonitrile -Butadiene-Styrene Blends, Polym. Int., 56 (2007) 1404-1414.

10. A. Nodera, T. Kanai, Flame Retardancy of Polycarbonate-Polydimethylsiloxane Block Copolymer/Silica Nanocomposites, J. Appl. Polym. Sci., 101 (2006) 3862-3868.

11. M. Iji, S. Serizawa, Silicone Derivatives as New Flame Retardants for Aromatic Thermoplastics Used in Electronic Devices, Polym. Adv. Technol., 9 (1998) 593-600. 
12. A. Genovese, R.A. Shanks, Fire Performance of Poly(dimethyl siloxane) Composites Evaluated by Cone Calorimetry, Composites Part A: applied science and manufacturing, 39 (2008) 398-405.

13. K. Chen, W. Zhou, J. Song. Preparation and Application of Polysiloxane Flame Retardant, China Plastics, 2009, 23 (2009) 87-90.

14. W. Zhou, H. Yang, Flame Retarding Mechanism of Polycarbonate Containing Methylphenyl-Silicone, Thermochim. Acta, 2007, 452 (2007) 43-48. 